\title{
Protocol for a systematic review on the evaluation of health literacy in adolescents and young adults with crohn's disease or type I diabetes mellitus and the relation with disease outcomes
}

\begin{abstract}
Background: Many children suffer from chronic diseases that greatly impactquality of life. As patients progress to adolescence and transition to adult care, health literacyshould be optimized. Health literacy is a crucial determinant of the ability to obtain, process and understand basic health information, make appropriate health decisions and interact effectively with health care professionals. Educational programs exist for some specific groups (e.g. diabetes).

Objectives and methods of study: This protocol describes the scope and methodology used for a systematic review of the health literacy of adolescents and young adults with Crohn's disease or diabetes mellitus type 1 who transitioned to adult health care. Results: The systematic review will explore the current knowledge on health literacy of the studied population and the relation with disease outcomes.
\end{abstract}

Conclusion: It is expected that this work provides a base for tailoring adequate educational programs for children and adolescents with Crohn's disease.

Keywords: health literacy, health knowledge, attitude, practise, inflammatory bowel diseases, crohn disease, diabetes mellitus, type1, adolescent, young adult, chronic disease
Volume 12 Issue 5 - 202I

\author{
Carels C, Levy E, Huysentruyt K, Veereman \\ G \\ Department of Paediatric Gastroenterology, Universitair \\ Ziekenhuis Brussel (UZ Brussel), Belgium
}

\author{
Correspondence: Veereman G, Department of Paediatric \\ Gastroenterology, Universitair Ziekenhuis Brussel (UZ Brussel), \\ Vrije Universiteit Brussel (VUB), Brussels, Belgium, \\ Email gveereman@gmail.com
}

Received: September 07, 2021 | Published: October 13, 2021
Abbreviations: BELCRO, paediatric belgian crohn's disease registry; $\mathrm{CD}$, crohn's disease; DM1, diabetes mellitus type 1; HL, health literacy; IBD, inflammatory bowel disease; QoL, quality of life; RQ, research question; SR, systematic review

\section{Background}

The purpose of this systematic review (SR) is to evaluate the health literacy (HL) of adolescents and young adults with Crohn's disease (CD) or diabetes mellitus type 1 (DM1) who transitioned to adult health care.

Many children suffer from chronic diseases that have a great impact on their quality of life. As these children grow up, they transition from a paediatric to adult health care system in their adolescence. In a paediatric health care system, the child depends on his or her parents, whereas the young adult should become independent and autonomous after transitioning. ${ }^{1,2}$

Adolescence is a period of increased vulnerability due to notable emotional and physical changes. During the transition to adultoriented health care, adolescents become responsible for their own health management. However, it is in this stage that health care utilisation often declines and that adolescents can be lost to follow-up care. ${ }^{3,4}$ If the transition is poorly managed, there is non-adherence to treatment with the risk ofworsening disease and emotional stress to the patient and his family. ${ }^{5}$ Therefore, it is important that adolescents are well prepared for the transition.

A criterionfor successful transition from one health care system to another is to master'the ability to obtain, process and understand basic health information, make appropriate health decisions and interact effectively with health care professionals', which is one of the definitions of HL. ${ }^{6}$ Thus, HL plays an important role in the outcome of a chronic disease.

$\mathrm{CD}$ is an inflammatory bowel disease (IBD) with important repercussions on daily life. Many patients are affected in their youth, which impacts on educational and professional choices. Guidelines for transition from paediatric to adult health care have been proposed. ${ }^{7}$ However, physicians do not routinely use validated tools to determine the patients' HL-related readiness for transition in IBD. ${ }^{6}$ IBD patients have a higher rate of nonparticipation in the work force and a tendency to take moresick leave, especially after surgery. ${ }^{8}$ The impact of IBD on work force participation is higher in $\mathrm{CD}$ than ulcerative colitis and in younger patients and those with higher education. ${ }^{9}$

A suitable population for comparison of the acquired HL are adolescents and young adults suffering from (juvenile-onset) DM1. High blood sugar levels due to defects in insulin secretion, insulin action, or both characterize this metabolic disorder. If left untreated, chronic hyperglycaemia can lead to long-term damage, dysfunction, and failure of different organs. The most affected organs are the eyes, nerves, kidneys, heart and blood vessels. ${ }^{10}$ Patients with low HL encounter difficulties to process and understand health information regarding their disease. ${ }^{11}$ Diabetics with poor health behaviours have a greater risk for numerous adverse health outcomes. ${ }^{12,13}$ Ferguson MO et al. demonstrated that a majority of patients with poorly controlled diabetes and with low HL believed that they were controlling their disease well or very well, which turned out not to be the case. Patients who incorrectly believe that they can manage their disease well are less likely to adapt their lifestyle to enhance control. ${ }^{14}$ On the other hand, patients with higher HL are less likely to smoke and control their 
glucose levels better. ${ }^{15}$ This is why HL is important on the individual level to manage the disease and the consequences. ${ }^{15}$

\section{Objectives}

The research question (RQ) for this SRis formulated as follows:

"What is the HL of adolescent and young adult patients with CD or DM1,

after they transitioned to adult care?"

A standardized PICO-based search strategy will be used:

a. P: Adolescents and young adults with $\mathrm{CD}$ or DM1, who transitioned to adult care

b. I $+\mathrm{C}$ : Evaluation of $\mathrm{HL}$ of a population

c. O: Disease related outcomes

\section{Methods}

The methodology used for this SR is based on the PRISMAstatement for elaboration of aSR (www.prisma-statement.org). A PRISMA-P 2015 checklist was completed. ${ }^{16}$

\section{Search strategy}

MEDLINE (Pubmed), Embase, Web of Science and Cochrane Central databases will be searched for original papers investigating the evaluation of HLof adolescents and young adults with CD or DM1 who transitioned to adult health care.

A structured search strategy will be used. We decided not to include the outcome in the search strategy to be sensitive enough and not too specific.

No language restrictions will be applied. One reviewer (CC) will also hand-search the references of included articles and other narrative and/or SRs to identify additional relevant publications. The literature search will include the following period: no limit for starting date September 2021 for end date.

The following search strategies will be used:

\section{Pubmed}

((((((“Adolescent”[Mesh]) OR “Young Adult”[Mesh]) OR “adolescen*”[TiAb]) OR “young adult*”[TiAb])) AND ((()(()(()(()((“Inflammatory Bowel Diseases”[Mesh]) OR “Crohn Disease"[Mesh]) OR IBD[TiAb]) OR crohn*[TiAb]) OR "crohn* disease"[TiAb]) OR "Diabetes Mellitus, Type 1"[Mesh]) OR "Diabetes Mellitus Type 1"[TiAb]) OR “Type 1 Diabetes"[TiAb]) OR "juvenile onset diabetes mellitus"[TiAb]) OR "juvenile-onset diabetes mellitus"[TiAb]) OR "juvenile-onset diabetes"[TiAb]) OR "juvenile onset diabetes"[TiAb]) OR "Chronic Disease"[Mesh]) OR "chronic disease*"[TiAb]) OR "chronic illness"”[TiAb]) OR "chronic condition*" [TiAb])) AND ((((("Health Literacy"[Mesh]) OR "Health Knowledge, Attitudes, Practice"[Mesh]) OR readability[TiAb]) OR literacy[TiAb]) OR knowledge[TiAb]).

\section{Embase}

('adolescent'/exp OR 'young adult'/exp OR 'adolescen*':ab,ti OR 'young adult*':ab,ti) AND ('inflammatory bowel disease'/ exp OR 'crohn disease'/exp OR 'ibd':ab,ti OR 'crohn*':ab,ti OR 'crohn* disease':ab,ti OR 'insulin dependent diabetes mellitus'/ exp OR 'insulin dependent diabetes mellitus':ab,ti OR 'diabetes mellitus type 1':ab,ti OR 'type 1 diabetes':ab,ti OR 'juvenile onset diabetes mellitus':ab,ti OR 'juvenile-onset diabetes mellitus':ab,ti
OR 'juvenile-onset diabetes':ab,ti OR 'juvenile onset diabetes':ab,ti OR 'chronic disease'/exp OR 'chronic disease*':ab,ti OR 'chronic illness*':ab,ti OR 'chronic condition*':ab,ti) AND ('health literacy'/ exp OR 'attitude to health'/exp OR readability:ab,ti OR literacy:ab,ti OR knowledge:ab,ti)

\section{Cochrane:}

\#1 MeSH descriptor: [Adolescent] explode all trees 95495

\#2 MeSH descriptor: [Young Adult] explode all trees 322

\#3 “adolescen*":ti,ab,kw (Word variations have been searched) 122388

\#4 “young adult*":ti,ab,kw (Word variations have been searched) 66837

$\# 5 \# 1$ or $\# 2$ or \#3 or \#4

158837

\#6 MeSH descriptor: [Inflammatory Bowel Diseases] explode all trees 2434

\#7 MeSH descriptor: [Crohn Disease] explode all trees

1179

\#8 IBD:ti,ab,kw (Word variations have been searched)

1152

\#9 crohn*:ti,ab,kw (Word variations have been searched) 3127

\#10 "crohn* disease":ti,ab,kw (Word variations have been searched) 2530

\#11 MeSH descriptor: [Diabetes Mellitus, Type 1] explode all trees 3940

\#12 “diabetes mellitus type 1":ti,ab,kw (Word variations have been searched) 4010

\#13 “type 1 diabetes":ti,ab,kw (Word variations have been searched) 3556

\#14 "juvenile onset diabetes mellitus":ti,ab,kw (Word variations have been searched) 4

\#15 “juvenile-onset diabetes mellitus”:ti,ab,kw (Word variations have been searched) 4

\#16 “juvenile-onset diabetes":ti,ab,kw (Word variations have been searched) 5

\#17 “juvenile onset diabetes":ti,ab,kw (Word variations have been searched) 5

\#18 MeSH descriptor: [Chronic Disease] explode all trees 12784

\#19 “chronic disease*":ti,ab,kw (Word variations have been searched) 17090

\#20 “chronic illness*":ti,ab,kw (Word variations have been searched) 1092

\#21 "chronic condition*":ti,ab,kw (Word variations have been searched) 1019

$\# 22 \# 6$ or $\# 7$ or $\# 8$ or \#9 or \#10 or \#11 or \#12 or \#13 or \#14 or \#15 or \#16 or \#17 or \#18 or \#19 or \#20 or \#21 28594

Citation: Carels C, Levy E, Huysentruyt K, et al. Protocol for a systematic review on the evaluation of health literacy in adolescents and young adults with crohn's disease or type I diabetes mellitus and the relation with disease outcomes. Gastroenterol Hepatol Open Access. 202 I; 2 (5): I4 I-I44. DOI: I0.15406/ghoa.202I.12.00475 

290

\#23 MeSH descriptor: [Health Literacy] explode all trees

\#24 MeSH descriptor: [Health Knowledge, Attitudes, Practice] explode all trees 5509

\#25 readability: ti,ab,kw (Word variations have been searched) 150

\section{7}

\#26 literacy: ti,ab,kw (Word variations have been searched)

\#27 knowledge: ti,ab,kw (Word variations have been searched) 24185

\#28 \#23 or \#24 or \#25 or \#26 or \#27 25290

\#29 \#5 and \#22 and \#28 233

\section{Web of science}

- $\mathrm{TS}=($ (“adolescen*" OR "young adult*") AND ("inflammatory bowel disease*" OR IBD OR crohn* OR "crohn* disease" OR "diabetes mellitus type 1" OR "type 1 diabetes" OR "juvenile onset diabetes mellitus" OR "juvenile-onset diabetes mellitus" OR "juvenile-onset diabetes" OR "juvenile onset diabetes" OR "chronic disease*" OR "chronic illness*" OR "chronic condition*") AND ("health literacy" OR readability OR literacy OR knowledge))

Indexes=SCI-EXPANDED, SSCI, A\&HCI, CPCI-S, CPCI-SSH, ESCI Timespan $=$ All years

\section{Study selection}

All observational and interventional studies and reviews reporting on the HL of a population with CD or DM1 will be included.

Exclusion criteria are:

1. Case reports and case series

2. (Meeting) abstract

3. Studies dealing exclusively with patients under 16years old and older than 24 years, or dealing with adolescents or young adults who did not transition to adult care

4. Studies on other chronic diseases

5. Studies dealing solely with the HL of caregiver or parent

6. Studies out of scope

Duplicates will be eliminated. A first selection will be done based on title and abstracts (CC,EL,GV and $\mathrm{KH}$ )and a second selection on a full text by 2 reviewers (EL,GV). Disagreements will be resolved by consensus. Endnote X9 software will be used to collect the articles.

\section{Risk of bias assessment, study appraisal}

All included studies will be scored independently by at least two reviewers $(\mathrm{EL}, \mathrm{GV}, \mathrm{KH})$ on their quality. The Newcastle Ottowa Scale will be used for prospective observational cohort studies.

\section{Data extraction and handling}

Three reviewers (EL,GV) will fill out a pre-designed Excel sheet to extract data including the following variables when available:

1. Name of the study

2. Year of the study
3. Type of study

4. Name of the country

5. Population size

6. Age of the population

7. Family history

8. Socio economic status

9. Type of disease

10. Duration of the disease

11. Treatment duration

12. Hl scale or questionnaire used

13. Results of the hl scale

14. Any measure of disease outcome (primary outcome)

15. Any data on qol (secondary outcome).

\section{Statistical analyses}

Analysis will be performed by KHand will be descriptive.We expect to find too many different measures for the assessment of HL to be able to perform a meta-analysis.

\section{Strengths and limitations}

Strengths of this SR are a very wide search of the literature on a subject with so far little information available. We expect that the results will help identify gaps in the evaluation of HL of adolescents and young adults and their care management.

Limitations of this SR are the poor use of standardized HL scores which will prevent comparison and statistical analysis of existing reports.

\section{Funding}

There is no funding for this work. The work will be part of the master thesis of one of the authors (CC).

\section{Author contributions}

a. CCwrote the protocol performed the literature search, and initiated study selection.

b. EL will perform further study selection and appraisal, data collection, data analysis, writing the manuscript and discussion.

c. KH participated in writing the protocol, literature search and study selection. He will perform study appraisal, data collection, data analysis.

d. GV supervised the process and participated in writing the protocol, study selection and willbe involved in study appraisal, data collection, data analysis and discussion.

e. The final manuscript will be reviewed and approved by all authors.

\section{Declaration of conflict of interests}

The authors have no conflict of interest to declare related to this work

Citation: Carels C, Levy E, Huysentruyt K, et al. Protocol for a systematic review on the evaluation of health literacy in adolescents and young adults with crohn's disease or type I diabetes mellitus and the relation with disease outcomes. Gastroenterol Hepatol Open Access. 202 I; I2(5): |4 I-I44. 


\section{Data statement}

The database including Endnote files, Excel files with study selection and extraction sheets will be available in a repository.

\section{References}

1. Abell RL, Winter M, Kreipe R, et al. Transition from Pediatric to Adult Centered Care: Lessons from the Literature and the Trenches. Adolesc Med State Art Rev. 2016;27(1):193-208.

2. Zijlstra M, De Bie C, Breij L, et al. Self-efficacy in adolescents with inflammatory bowel disease: a pilot study of the "IBD-yourself", a disease-specific questionnaire. J Crohns Colitis. 2013;7(9):e375-e385.

3. Beal SJ, lka K Riddle IK, Kichler JC, et al. The Associations of Chronic Condition Type and Individual Characteristics With Transition Readiness. Acad Pediatr. 2016;16(7):660-667.

4. d'Emden H, McDermott B, D'Silva N, et al. Psychosocial screening and management of young people aged 18-25years with diabetes. Intern Med J. 2017;47(4):415-423.

5. Gray WN, Resmini AR, Baker KD, et al. Concerns, Barriers, and Recommendations to Improve Transition from Pediatric to Adult IBD Care: Perspectives of Patients, Parents, and Health Professionals. Inflamm Bowel Dis. 2015;21(7):1641-1651.

6. Huang JS, Tobin A, Tompane T, et al. Clinicians poorly assess health literacy-related readiness for transition to adult care in adolescents with inflammatory bowel disease. Clin Gastroenterol Hepatol. 2012;10(6):626632.

7. NICE. Transition: Overarching principles. NICE guideline [NG43].2016.
8. Marri SR, Buchman AL. The education and employment status of patients with inflammatory bowel diseases. Inflamm Bowel Dis. 2005;11(2):171177.

9. Boonen A, Dagnelie PC, FeleusA, et al. The impact of inflammatory bowel disease on labor force participation: results of a population sampled case-control study. Inflamm Bowel Dis. 2002;8(6):382-389.

10. Association AD. Diagnosis and classification of diabetes mellitus. Diabetes Care. 2010;33 Suppl 1:S62-S69.

11. Stiles E. Promoting health literacy in patients with diabetes. Nurs Stand. 2011;26(8):35-40.

12. Friis K, Vind BD, Simmons RK, et al. The Relationship between Health Literacy and Health Behaviour in People with Diabetes: A Danish Population-Based Study. J Diabetes Res. 2016;2016:7823130.

13. Iris van der H, Uiters E, Rademakers $J$, et al. Associations among health literacy, diabetes knowledge, and self-management behavior in adults with diabetes: results of a dutch cross-sectional study. J Health Commun. 2014;19 Suppl 2:115-131.

14. Ferguson MO, Long JA, Zhu J, et al. Low Health Literacy Predicts Misperceptions of Diabetes Control in Patients With Persistently Elevated A1C. Diabetes Educ. 2015 Jun;41(3):309-319.

15. Dorner TE, Lackinger C, Schindler $\mathrm{K}$, et al. Health information regarding diabetes mellitus reduces misconceptions and underestimation of consequences in the general population. Public Health Nut. 2013;16(11):2032-2039.

16. Moher D, Liberati A, Tetzlaff J, et al. Preferred reporting items for systematic reviews and meta-analyses: the PRISMA statement. PLoS Med. 2009;6(7):e1000097. 